\title{
Editorial
}

\section{JNC 8 and Need for Our Own Hypertension Guideline}

\author{
M Zahirul Haque ${ }^{1}$
}

Recently the Eighth Joint National Committee (JNC 8) has released its new guidelines on the management of adult hypertension, which contain two key changes from the previous JNC 7 that will simplify the care.

First, JNC 8 recommends a relaxing of the more aggressive JNC 7 target blood pressures and treatmentinitiation thresholds in elderly patients and in patients under age 60 with diabetes and kidney disease. JNC 8 also backs away from the recommendation that thiazide-type diuretics should be initial therapy in most patients, suggesting an ACE inhibitor, angiotensinreceptor blocker (ARB), calcium-channel blocker (CCB), or thiazide-type diuretic are reasonable choices.

A condensed version of the guideline's nine recommendations is as follows-

In the general population ages 60 and older, pharmacologic treatment to lower BP should be initiated at a systolic blood pressure (SBP) of 150 $\mathrm{mmHg}$ or higher or a diastolic blood pressure (DBP) of $90 \mathrm{mmHg}$ or higher. In the general population younger than age 60, initiate pharmacologic treatment at a DBP of $90 \mathrm{mmHg}$ or higher or an SBP of $140 \mathrm{mmHg}$ or higher and treat to goals below these respective thresholds.

In the population ages 18 years or older with diabetes or CKD, initiate pharmacologic treatment at an SBP of $140 \mathrm{mmHg}$ or higher or a DBP of $90 \mathrm{mmHg}$ or higher and treat to goals below these respective thresholds.

In the general nonblack population, including those with diabetes, initial treatment should include a thiazide-type diuretic, calcium channel blocker (CCB), angiotensin-converting enzyme (ACE) inhibitor or angiotensin receptor blocker (ARB).

In the general black population, including those with diabetes, initial treatment should include a thiazide-type diuretic or a CCB.

In the population ages 18 or older with CKD and hypertension, initial (or add-on) treatment should include an ACE inhibitor or an ARB to improve kidney outcomes. This applies to all patients in this population regardless of race or diabetes status.

If goal BP is not reached within a month of initiating treatment, increase the dose of the initial drug or add a second drug from one of these four classes. If goal BP cannot be reached with two drugs, add and titrate a third drug from the list provided.

ACE inhibitors and ARBs should not be used concomitantly. If goal BP cannot be reached using the above-named drugs because of a contraindication or the need to use more than three such drugs to reach goal $\mathrm{BP}$, antihypertensive drugs from other classes may be used.

There is a rising prevalence of hypertension in the Bangladeshi population and increase in cardiovascular disease. We know that Bangladeshis are racially different from others and the risk factors for cardiovascular disease defined for us are different from those for other populations, such as Caucasians or Africans, as we are more prone to heart disease. We also need our own guidelines in view of our special geographical, ethnic, and climatic conditions and very different dietary habits. 